\title{
A New Parallel Difference Numerical Method for the Payment of Dividend Black- Scholes Equation
}

\author{
Xiaozhong Yang, Fan Zhang \\ Dept. Mathematics and physics, North China Electric Power University \\ Beijing, 102206, China. \\ yxiaozh@ncepu.edu.cn,nmsdzf90@163.com.
}

\begin{abstract}
A new alternating segment explicit-implicit and alternating segment implicit-explicit methods for solving the payment of dividend Black-Scholes equation are presented. These new methods have several advantages such as: good parallelism, unconditional stability, convergence and better accuracy. Numerical experiments show that the methods improve the calculation speed greatly.
\end{abstract}

Keywords-payment of dividend Black-Scholes equation; alternating segment explicit-implicit scheme; parallel computing; numerical experiment

\section{INTRODUCTION}

The option is one of the most important financial derivatives which has an extremely significant position in the modern finance activity[1]. Although the European option has analytic expression, it cannot meet the effective requirement as the form of the expression is too complex to calculate quickly. In practice, we usually use the numerical method instead, such as the finite difference numerical method. Lifei $\mathrm{Wu}$ and Xiaozhong Yang(2010) first put forward the 'explicit-implicit' and 'implicit-explicit' difference schemes for solving the payment of dividend Black-Scholes equation[3]; Xiaozhong Yang and Yangguo Liu(2007) proposed the general difference scheme for solving the Black-Scholes equation[5]. However, most of the schemes are calculated in serial way and the efficiency is very low.

So far, the numerical solution method for the option pricing problems is divided into two types: explicit method and implicit method. As we know, the explicit method has features of simple calculation but poor stability and low precision; the implicit method is well stability, high precision but not suitable for parallel computing[4]. With the rapid development of multi-core and cluster technology, parallel programming becomes one of the mainstream technology for improving the calculation efficiency. Therefore, construct the difference scheme with high stability and intrinsic parallelism is considerable important from the scientific and practical views. This paper gives the parallel difference schemes for solving the payment of dividend Black-Scholes equation: the alternating explicit-implicit scheme and the alternating implicit-explicit scheme. Meanwhile, this paper also analyzes the stability, convergence and computing economy. Finally, some numerical examples verify the effectiveness of these schemes.

\section{Alternating SEgMENT EXPLICIT-IMPLICIT SCHEME}

A. The payment of dividend Black-Scholes equation

Assume that the underlying asset is the dividend-paying stock, by the $\Delta$-hedging principle, we can get the following equation[1,2]:

$$
\left\{\begin{array}{c}
\frac{\partial V}{\partial t}+\frac{\sigma^{2}}{2} S^{2} \frac{\partial^{2} V}{\partial S^{2}}+(r-q) S \frac{\partial V}{\partial S}-r V=0 \\
V(S, T)=\max \{S-K, 0\}
\end{array}\right.
$$

In theory, the solving area of this equation is:

$$
\Omega=\left\{(S, t) \mid S_{\min }<S<S_{\max }, t \in[0, T]\right\}
$$

In order to solve Eq.(1), we can substitute its variables as follows:

$$
S=e^{x}, t=T-\tau, V(S, t)=U(x, \tau)
$$

Then this pricing model will be transformed into the initialboundary value problem of partial differential equation with constant coefficients:

$$
\left\{\begin{array}{c}
\frac{\partial U}{\partial t}-\frac{\sigma^{2}}{2} \frac{\partial^{2} U}{\partial x^{2}}-\left(r-q-\frac{\sigma^{2}}{2}\right) \frac{\partial U}{\partial x}+r U=0 \\
U(x, 0)=\max \left\{e^{x}-K, 0\right\}
\end{array}\right.
$$

\section{B. Alternating Segment Explicit-Implicit Scheme}

Make a mesh partition on the area $\Omega$, let $h$ as the space step and $\mathrm{k}$ as the time step, and the number of grid points are $(m+1) \times(n+1), r_{1}=k / h^{2}$. We use $U_{i}^{j}$ to denote the finite difference approximations of $U\left(x_{i}, \tau_{j}\right)$. In order to construct the alternating segment explicit-implicit scheme, we give some difference schemes of the Eq. (2).

First, the classical explicit scheme[8].

$$
\begin{aligned}
\frac{U_{i}^{j+1}-U_{i}^{j}}{k}= & \frac{\sigma^{2}}{2} \frac{U_{i+1}^{j}-2 U_{i}^{j}+U_{i-1}^{j}}{h^{2}}+\left(r-q-\frac{\sigma^{2}}{2}\right) \\
& \frac{U_{i+1}^{j}-U_{i-1}^{j}}{2 h}-r U_{i}^{j}
\end{aligned}
$$

That is: 


$$
\begin{aligned}
U_{i}^{j+1}= & {\left[\frac{\sigma^{2}}{2} r_{1}-\left(r-q-\frac{\sigma^{2}}{2}\right) \frac{k}{2 h}\right] U_{i-1}^{j}+\left(1-\sigma^{2} r_{1}\right.} \\
& -r k) U_{i}^{j}+\left[\frac{\sigma^{2}}{2} r_{1}+\left(r-q-\frac{\sigma^{2}}{2}\right) \frac{k}{2 h}\right] U_{i+1}^{j}
\end{aligned}
$$

Then, give the classical implicit scheme[8].

$$
\begin{aligned}
U_{i}^{j}=[ & \left.-\frac{\sigma^{2}}{2} r_{1}+\left(r-q-\frac{\sigma^{2}}{2}\right) \frac{k}{2 h}\right] U_{i-1}^{j+1}+\left(1+\sigma^{2} r_{1}\right. \\
& +r k) U_{i}^{j+1}-\left[\frac{\sigma^{2}}{2} r_{1}+\left(r-q-\frac{\sigma^{2}}{2}\right) \frac{k}{2 h}\right] U_{i+1}^{j+1}
\end{aligned}
$$

At last, we present the improved Saul'yev asymmetric scheme. In order to meet the parallelism of the difference equation, we construct two improved Saul'yev asymmetric schemes of Eq.(2) as follows:

$$
\begin{aligned}
& \left(1+\frac{\sigma^{2}}{2} r_{1}+\frac{r}{2} k\right) U_{i}^{j+1}-\left[\frac{\sigma^{2}}{2} r_{1}+\left(r-q-\frac{\sigma^{2}}{2}\right) \frac{k}{2 h}\right] U_{i+1}^{j+1} \\
& =\left(1-\frac{\sigma^{2}}{2} r_{1}-\frac{r}{2} k\right) U_{i}^{j}+\left[\frac{\sigma^{2}}{2} r_{1}-\left(r-q-\frac{\sigma^{2}}{2}\right) \frac{k}{2 h}\right] U_{i-1}^{j} \\
& \left(1+\frac{\sigma^{2}}{2} r_{1}+\frac{r}{2} k\right) U_{i}^{j+2}-\left[\frac{\sigma^{2}}{2} r_{1}-\left(r-q-\frac{\sigma^{2}}{2}\right) \frac{k}{2 h}\right] U_{i-1}^{j+2} \\
& =\left(1-\frac{\sigma^{2}}{2} r_{1}-\frac{r}{2} k\right) U_{i}^{j+1}+\left[\frac{\sigma^{2}}{2} r_{1}+\left(r-q-\frac{\sigma^{2}}{2}\right) \frac{k}{2 h}\right] U_{i+1}^{j+1}
\end{aligned}
$$

Then give the accuracy analysis of the scheme. Make difference between the two sides of Eq.(5) and Eq.(6), and we will get the truncation error:

$$
\begin{aligned}
R_{i}^{j+1}= & \frac{k^{2}}{6} \frac{\partial^{3} U}{\partial \tau^{3}}-\frac{\sigma^{2}}{2}\left(\frac{k}{h} \frac{\partial^{2} U}{\partial x \partial \tau}-\frac{k^{2}}{2 h} \frac{\partial^{3} U}{\partial x \partial \tau^{2}}\right)-(r-q- \\
& \left.\frac{\sigma^{2}}{2}\right)\left(\frac{k}{2 h} \frac{\partial U}{\partial \tau}-\frac{k^{2}}{4 h} \frac{\partial^{2} U}{\partial \tau^{2}}+\frac{h^{2}}{6} \frac{\partial^{3} U}{\partial x^{3}}+\frac{1}{4} k h \frac{\partial^{3} U}{\partial x^{2} \partial \tau}\right. \\
& \left.+\frac{k^{2}}{4} \frac{\partial^{3} U}{\partial x \partial \tau^{2}}+\frac{k^{3}}{12 h} \frac{\partial^{3} U}{\partial \tau^{3}}\right)+\left(\frac{k^{2}}{4} \frac{\partial^{2} U}{\partial \tau^{2}}-\frac{k^{3}}{12} \frac{\partial^{3} U}{\partial \tau^{3}}\right) r \\
& +o\left(k^{\alpha} h^{\beta}\right)
\end{aligned}
$$

$$
\begin{aligned}
R_{i}^{j+2}= & \frac{k^{2}}{6} \frac{\partial^{3} U}{\partial \tau^{3}}-\frac{\sigma^{2}}{2}\left(-\frac{k}{h} \frac{\partial^{2} U}{\partial x \partial \tau}-\frac{k^{2}}{2 h} \frac{\partial^{3} U}{\partial x \partial \tau^{2}}\right)-(r-q- \\
& \left.\frac{\sigma^{2}}{2}\right)\left(-\frac{k}{2 h} \frac{\partial U}{\partial \tau}-\frac{k^{2}}{4 h} \frac{\partial^{2} U}{\partial \tau^{2}}+\frac{h^{2}}{6} \frac{\partial^{3} U}{\partial x^{3}}-\frac{1}{4} k h \frac{\partial^{3} U}{\partial x^{2} \partial \tau}\right. \\
& \left.+\frac{k^{2}}{4} \frac{\partial^{3} U}{\partial x \partial \tau^{2}}-\frac{k^{3}}{12 h} \frac{\partial^{3} U}{\partial \tau^{3}}\right)+\left(\frac{k^{2}}{4} \frac{\partial^{2} U}{\partial \tau^{2}}+\frac{k^{3}}{12} \frac{\partial^{3} U}{\partial \tau^{3}}\right) r \\
& +o\left(k^{\alpha} h^{\beta}\right)
\end{aligned}
$$

Here $\alpha+\beta=4$. As the alternative use of the scheme (5) and scheme (6) at different time interval, parts of the error terms in the two truncation error expressions can be counterbalanced. Therefore we will get the following theorem:

Theorem 1: The calculation accuracy of the improved Saul'yev asymmetric scheme (5) and (6) of the dividendpaying option pricing is $O\left(k^{2}+h^{2}+k^{2} / h\right)$.

Among the schemes mentioned above, the classic explicit scheme (3) has the nature property of parallelism and is very suitable for parallel computing, but it is conditionally stable; the classic implicit scheme (4) is stable unconditional, but it needs to solve an algebraic equation which cannot be implemented on parallel computer.

The alternating explicit-implicit is constructed based on the combined use of the schemes mentioned above, the design is as follows:

Let $m-1=N l$, here $N$ is a positive odd number, $l$ is a positive integer, $N, l \geq 3$, we divide the points on each time level into $N$ sections. And on the even level, we arrange the computation according to the rule of 'the explicit segmentthe implicit segment- $\cdots$-the explicit segment'[7]. For realize the parallel computing, the left endpoint of the implicit segment is calculated with the improved Saul'yev scheme (5), the right endpoint is calculated with the improved Saul'yev scheme (6). When it turns to the odd level, the rule changes into 'the implicit segment-the explicit segment-... the implicit segment', then the alternating segment explicitimplicit scheme can be expressed as

$$
\begin{aligned}
& \left(I+r_{1} G_{1}\right) U^{j+1}=\left(I-r_{1} G_{2}\right) U^{j}+b^{j} \\
& \left(I+r_{1} G_{2}\right) U^{j+2}=\left(I-r_{1} G_{1}\right) U^{j+1}+b^{j+1}
\end{aligned}
$$


$\begin{aligned} G_{1} & =\left(\begin{array}{cccccc}Q_{l} & & & & & \\ & G_{l}^{(1)} & & & & \\ & & Q_{l} & & & \\ & & & \ddots & & \\ & & & & G_{l}^{\left(\frac{N-1}{2}\right)} & \\ & & & & & Q_{l}\end{array}\right) \\ G_{2} & =\left(\begin{array}{cccccc}\bar{G}_{l+1}^{(1)} & & & & & \\ & Q_{l-2} & & & & \\ & & G_{l+2}^{(2)} & & & \\ & & & \ddots & & \\ & & & & Q_{l-2} & \\ & & & & & \bar{G}_{l+1}^{\left(\frac{N+1}{2}\right)}\end{array}\right)\end{aligned}$

$\bar{G}_{l+1}^{(1)}=\left(\begin{array}{ccccc}2 b_{1} & -c_{1} & & & \\ -a_{1} & 2 b_{1} & -c_{1} & & \\ & \ddots & \ddots & \ddots & \\ & & -a_{1} & 2 b_{1} & -c_{1} \\ & & & -a_{1} & b_{1}\end{array}\right)_{(I+1) \times(l+1)}$

$G_{l^{\prime}}^{(i)}=\left(\begin{array}{ccccc}b_{1} & -c_{1} & & & \\ -a_{1} & 2 b_{1} & -c_{1} & & \\ & \ddots & \ddots & \ddots & \\ & & -a_{1} & 2 b_{1} & -c_{1} \\ & & & -a_{1} & b_{1}\end{array}\right)_{l^{\prime} \times l^{\prime}}$

$\bar{G}_{I+1}^{\left(\frac{N+1}{2}\right)}=\left(\begin{array}{ccccc}2 b_{1} & -c_{1} & & & \\ -a_{1} & 2 b_{1} & -c_{1} & & \\ & \ddots & \ddots & \ddots & \\ & & -a_{1} & 2 b_{1} & -c_{1} \\ & & & -a_{1} & 2 b_{1}\end{array}\right)_{(I+1) \times(l+1)}$

$a_{1}=\frac{\sigma^{2}}{2}-\left(r-q-\frac{\sigma^{2}}{2}\right) \frac{h}{2} ; b_{1}=\frac{\sigma^{2}}{2}+\frac{r}{2} h^{2}$ $c_{1}=\frac{\sigma^{2}}{2}+\left(r-q-\frac{\sigma^{2}}{2}\right) \frac{h}{2} ; l^{\prime}=l$ or $l+2 ; Q_{l}$ is the zero matrix.

C. Stability and convergence analysis

By eliminating $U^{j+1}$ from Eq.(7), we obtain

in which

$$
U^{j+2}=T U^{j}+b^{\prime}
$$

$$
T=\left(I+r_{1} G_{2}\right)^{-1}\left(I-r_{1} G_{1}\right)\left(I+r_{1} G_{1}\right)^{-1}\left(I-r_{1} G_{2}\right)
$$

For stability it is necessary that $\|T\| \leq 1$. In proving the stability condition, the following lemma Kellogg is used.

Lemma 1[6]: If $\rho>0$ and $\left(C+C^{T}\right)$ is non-negative (or positive) define, then

$$
\left\|(\rho I-C)(\rho I+C)^{-1}\right\|_{2} \leq 1 .
$$

Now define the matrix $\widetilde{T}$ as follows:

$$
\widetilde{T}=\left(I+r_{1} G_{2}\right) T\left(I+r_{1} G_{2}\right)^{-1}
$$

which is similar to $T$ and thus has the same eigenvalues as T. With using Eq.(8), $\widetilde{T}$ can be expressed as

$$
\widetilde{T}=\left(I-r_{1} G_{1}\right)\left(I+r_{1} G_{1}\right)^{-1}\left(I-r_{1} G_{2}\right)\left(I+r_{1} G_{2}\right)^{-1} .
$$

Therefore we have

$$
\begin{aligned}
\|T\|_{2} & =\|\widetilde{T}\|_{2} \\
& \leq\left\|\left(I-r_{1} G_{1}\right)\left(I+r_{1} G_{1}\right)^{-1}\right\|_{2}\left\|\left(I-r_{1} G_{2}\right)\left(I+r_{1} G_{2}\right)^{-1}\right\|_{2}
\end{aligned}
$$

Since $\left(G_{1}+G_{1}^{T}\right)$ and $\left(G_{2}+G_{2}^{T}\right)$ are non-negative, definite form (9) and Lemma 1, the unconditional stability condition is obtained for scheme governed by (7) for all $r_{1}>0$. Hence one can state the following theorem.

Theorem 2: The parallel implementation of alternating segment explicit-implicit scheme (7) for the solution of the dividend-paying option pricing equation (2) is unconditional stable.

By Lax Theorem[4], we can also obtain the following corollary.

Corollary 1: The parallel implementation of alternating segment explicit-implicit scheme (7) for the solution of dividend-paying option pricing equation (2) is convergent.

Similarly, if we adopt the rule of 'the implicit segmentthe explicit segment- $\cdots$-the implicit segment' on the even level, and adopt the rule of ' the explicit segment-the implicit segment- $\cdots$-the explicit segment' on the odd level, we can construct the alternating implicit-explicit scheme as follows:

$$
\begin{aligned}
& \left(I+r_{1} G_{2}\right) U^{j+1}=\left(I-r_{1} G_{1}\right) U^{j}+b^{j} \\
& \left(I+r_{1} G_{1}\right) U^{j+2}=\left(I-r_{1} G_{2}\right) U^{j+1}+b^{j+1}
\end{aligned}
$$

And if we apply the same method on the alternating implicit-explicit scheme (10), we will get the similar theorem. Theorem 3: The parallel implementation of alternating segment implicit-explicit scheme (10) for the solution of 
dividend-paying option pricing equation (2) is unconditional stable and convergent.

\section{NUMERICAL EXPERIMENTS}

Here, we consider a European call option on stocks with dividends. Assuming the strike price of option is 90 dollars, the risk-free interest rate is 0.06 per year, the dividend rate is 0.01 per year, the volatility is 0.3 . Consider the deadline of the option is 3 and 6 months[2].

The numerical experiment is done in Matlab R2009 environment[9]. The comparison among the analytic solution and numerical solutions, such as the results of the alternating segment explicit-implicit scheme (ASE-I), the explicitimplicit scheme and the Crank-Nicolson scheme is shown in the following table and the comparison of the parallel calculation time and the serial calculation time is shown in the following figure.

TABLE I. COMPARISON OF SEVERAL SCHEMES' RESULTS WITH THE ANALYTIC SOLUTION.

\begin{tabular}{|c|c|c|c|}
\hline The Schemes & 3 months(\$) & $\mathbf{6}$ months(\$) & Relative errors \\
\hline Analytic solution $^{[1]}$ & 5.909983 & 8.628140 & \\
\hline Crank-Nicolson $^{[5]}$ & 5.910371 & 8.628440 & $0.0065 \%$ \\
\hline Explicit-implicit $^{[3]}$ & 5.910323 & 8.628148 & $0.0057 \%$ \\
\hline Implicit-explicit $^{[3]}$ & 5.910323 & 8.628148 & $0.0057 \%$ \\
\hline ASE-I & 5.910328 & 8.628431 & $0.0058 \%$ \\
\hline ASI-E & 5.910328 & 8.628431 & $0.0058 \%$ \\
\hline
\end{tabular}

Then we compare these schemes' calculation time, the result is shown in the following figure.

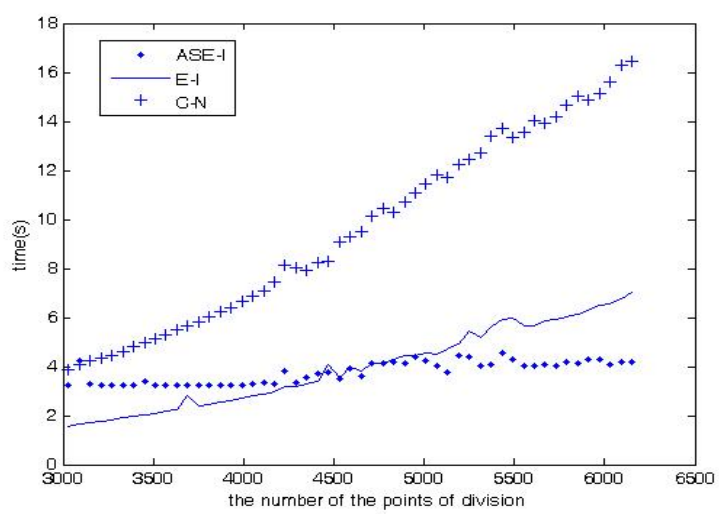

Figure 1. The comparison of some schemes' calculation time
From the table and the figure of the comparison among analytic solution and numerical solution of several schemes above, we can see the alternating segment explicit-implicit has the similar calculation accuracy with the explicit-implicit, but the calculation time is $1 / 2$ of that of the explicit-implicit scheme and $1 / 5$ of that of the Crank-Nicolson scheme. Thus the scheme given by this paper improves the calculation speed greatly and has greater advantage in solving the option pricing problem.

\section{ACKNOWLEDGMENT}

This work has been supported by the National Science Foundation of China under Grant No.10771065, the key project of foreign experts Chinese Ministry of Education (2010) and Special Funds for Co-construction Project of Beijing.

\section{REFERENCES}

[1] Lishang Jiang, Mathematical Modeling and Models of Option Pricing (Second Edition), Higher Education Press, Beijing, 2008. (in Chinese)

[2] Shengmin Zhao, Finance Derivative Tools Pricing, China Financial and Economic Publishing House, Beijing, 2008. (in Chinese)

[3] Xiaozhong Yang, Lifei Wu, "Explicit-implicit and Implicit-explicit Algorithm for Solving the Payment of Dividend Black-Scholes Equation," Highlights of Science Paper Online, vol.4, pp.1208-1211, 2007.

[4] Baolin Zhang, Guoxing Yuan, The Parallel Finite Difference Method for the Partial Difference Equation, Science Press, Beijing, 1994. (in Chinese)

[5] Xiaozhong Yang, Yangguo Liu, "Study on a New Kind of Universal Difference Schemes for Solving Black-Scholes Equation," International Journal of Numerical Analysis and Modeling, vol.3, pp.251-256, 2007.

[6] D.J.Evans, A.R.B.Abdullan, "Group Explicit Method for Parabolic Equation,” Int.J. Computer Math, vol.14, pp.73-105, 1983.

[7] Jinfu Lu, Baolin Zhang, and Tao Xu, "The Alternating Segment Explicit-implicit Method for Solving the Convection-diffusion equation," Numerical Methods and Computer Applications, vol.3, pp.162-167, 1988.

[8] Suochun Zhang, Finite Difference Numerical Calculation for Parabolic Equation with Boundary Condition, Science Press, Beijing, 2010. (in Chinese)

[9] Wei Liu, Actual Combating Matlab of Parallel Computing, Beijing University of Aeronautics and Astronautics Publishing House, Beijing, 2012. (in Chinese) 